\title{
ON THE LENGTH OF AN EXTERNAL BRANCH IN THE BETA-COALESCENT
}

\author{
JEAN-STÉPHANE DHERSIN, FABIAN FREUND, ARNO SIRI-JÉGOUSSE, AND LINGLONG YUAN
}

\begin{abstract}
In this paper, we consider $\operatorname{Beta}(2-\alpha, \alpha)$ (with $1<\alpha<2)$ and related $\Lambda$ coalescents. If $T^{(n)}$ denotes the length of an external branch of the $n$-coalescent, we prove the convergence of $n^{\alpha-1} T^{(n)}$ when $n$ tends to $\infty$, and give the limit. To this aim, we give asymptotics for the number $\sigma^{(n)}$ of collisions which occur in the $n$-coalescent until the end of the chosen external branch, and for the block counting process associated with the $n$-coalescent.
\end{abstract}

\section{INTRODUCTION}

1.1. Motivation and main results. In modern genetics, it is possible to sequence whole genomes of individuals. In order to put this information to maximal use, it is important to have well-fitting models for the gene genealogy of a sample of individuals. The standard model for a gene genealogy of a sample of $n$ individuals is Kingman's $n$-coalescent (see [26], [27]). Kingman's $n$-coalescent is a continuous-time Markov process with state space $\mathcal{P}^{(n)}$, the set of partitions of $\{1, \ldots, n\}$. The process starts in the trivial partition $(\{1\}, \ldots,\{n\})$ and transitions are only possible as mergers of exactly two blocks of the current state. Each such binary merger occurs with rate 1 . These mergers are also called collisions.

For many populations, Kingman's $n$-coalescent describes the genealogy quite well. Kingman showed in [27] that the ancestral trees of a sample of size $n$ in populations with size $N$ evolving by a Wright-Fisher model will converge weakly to Kingman's $n$-coalescent for $N \rightarrow \infty$ (after a suitable time-change). This result is relatively robust if population evolution deviates from the Wright-Fisher model (see [27] or [28]). However, there is evidence that there are populations where the gene genealogy of a sample is not described well by Kingman's $n$-coalescent. Examples of such populations can be found in maritime species, where one individual can have a huge number of offspring with non-negligible probability (see [1], [12] [22], [20] and [16]).

A whole class of potential models for the gene genealogy of a sample was introduced independently by Pitman and Sagitov (see [30] and [31]): The class of $n$-coalescents with multiple collisions. A $n$-coalescent with multiple collisions is a continuous-time Markov process with state space $\mathcal{P}^{(n)}$, where all possible transitions are done by merging two or more blocks of the current state into one new block. Every $n$-coalescent $\Pi^{(n)}$ is exchangeable, meaning $\tau \circ \Pi^{(n)} \stackrel{d}{=} \Pi^{(n)}$ for every permutation $\tau$ of $\{1, \ldots, n\}$. The transition rate of a merger/collision of $k$ of $b$ present blocks is given by

$$
\lambda_{b, k}=\int_{0}^{1} x^{k}(1-x)^{b-k} x^{-2} \Lambda(d x)
$$

Date: January 17, 2012.

2010 Mathematics Subject Classification. 60J70, 60J80, 60J25, 60F05, 92D25.

Key words and phrases. Coalescent process, Beta-coalescent, external branch, block counting process, recursive construction. 
for a finite measure $\Lambda$ on $[0,1]$ (this definition is due to Pitman [30]). Since the process is characterized by the measure $\Lambda$, it is also called a $\Lambda$ - $n$-coalescent. Note that Kingman's $n$-coalescent is a $\Lambda$ - $n$-coalescent with $\Lambda$ being the Dirac measure $\delta_{0}$ in 0 .

It is possible to define a continuous-time process $\Pi$ with state space $\mathcal{P}$, the partitions of the natural numbers $\mathbb{N}$, whose restriction on $\{1, \ldots, n\}$ is a $\Lambda$ - $n$-coalescent for all $n \in \mathbb{N}$. Such a process is called a $\Lambda$-coalescent.

An important subclass of $\Lambda$ - $n$-coalescents are Beta $n$-coalescents characterized by $\Lambda$ being a Beta distribution, especially for the choice of parameters $2-\alpha$ and $\alpha$ for $\alpha \in(0,2)$. The class of $\operatorname{Beta}(2-\alpha, \alpha)$-n-coalescents appears as ancestral trees in various settings. They appear in the context of supercritical Galton-Watson processes (see [32]), of continuous-state branching processes (see [9]) and of continuous random trees (see [3]). They also seem to be a class where suitable models for the ancestral tree can be found for samples from species who do not fit well with the Kingman-setting (see [8]). Note that for $\alpha \rightarrow 2$, the rates of the $\operatorname{Beta}(2-\alpha, \alpha)$ - $n$-coalescent converge to the rates of Kingman's $n$-coalescent. In this sense, Kingman's $n$-coalescent can be seen as a border case of this class of Beta $n$-coalescents.

For $\alpha=1, \operatorname{Beta}(2-\alpha, \alpha)$ is the uniform distribution on $[0,1]$. The corresponding $n$ coalescent is the Bolthausen-Sznitman $n$-coalescent. It appears in the field of spin glasses (see [11], [13]) and is also connected to random recursive trees (see [21]).

Let us denote by $\Pi^{(n)}=\left(\Pi_{t}^{(n)}\right)_{t \geq 0}$ a $n$-coalescent. In this paper, we are interested in three functionals of $n$-coalescents

- the length $T^{(n)}$ of a randomly chosen external branch ;

- the number $\sigma^{(n)}$ of collisions which occur in the $n$-coalescent until the end of a randomly chosen external branch ;

- the block counting process $R^{(n)}=\left(R_{t}^{(n)}\right)_{t \geq 0}: R_{t}^{(n)}=\left|\Pi_{t}^{(n)}\right|$ is the number of blocks of $\Pi_{t}^{(n)}$.

Note that $T^{(n)}$ can also be characterized as the waiting time for the first collision of a randomly chosen individual and $\sigma^{(n)}$ as the number of collisions we have to wait to see the randomly chosen individual merge. For $i \in\{1, \ldots, n\}$ define

$$
T_{i}^{(n)}:=\inf \left\{t \mid\{i\} \notin \Pi_{t}^{(n)}\right\}
$$

as the length of the $i$ th external branch and

$$
\sigma_{i}^{(n)}:=\inf \left\{k \mid\{i\} \notin \pi_{k}\right\}
$$

as the number of collisions until the end of the $i$ th external branch, where $\pi_{k}$ is the state of the $n$-coalescent after $k$ jumps. Due to the exchangeability of the $n$-coalescent, we have $T^{(n)} \stackrel{d}{=} T_{1}^{(n)}$ and $\sigma^{(n)} \stackrel{d}{=} \sigma_{1}^{(n)}$. Since we are only interested in distributional results, for the remainder of the article we will identify $T^{(n)}$ with $T_{1}^{(n)}$ and $\sigma^{(n)}$ with $\sigma_{1}^{(n)}$.

If the $n$-coalescent is used as a model for an ancestral tree of a sample of individuals/genes, the functionals $T^{(n)}$ and $\sigma^{(n)}$ can be interpreted biologically. The length of an external branch measures the uniqueness of the individual linked to that branch compared to the sample, since it gives the time this individual has to evolve by mutations that do not affect the rest of the sample (see the introduction of [14] for more information). It was first introduced by Fu and $\mathrm{Li}$ in [18], where they compare mutations on external and internal branches of Kingman's $n$-coalescent in order to test for the neutrality of mutations. 
The functional $\sigma^{(n)}$ was first introduced in [14], though $n-\sigma^{(n)}$ was also analyzed in [10] as the level of coalescence of the chosen individual with the rest of the sample. In both articles, the functionals were defined for Kingman's $n$-coalescent.

For the biological interpretation of $\sigma^{(n)}$, we see the $n$-coalescent as an ancestral tree of a sample of size $n$. Each collision in the $n$-coalescent then resembles the emergence of an ancestor of the sample. $\sigma^{(n)}-1$ is the number of ancestors of the sample which emerge before the most recent ancestor of the randomly chosen individual/gene emerges (recall that time runs backwards in the $n$-coalescent). In this line of thought, $\sigma^{(n)}$ gives the temporal position of the first ancestor of the chosen individual/gene among all ancestors of the sample, which are the $\tau^{(n)}$ collisions in the $n$-coalescent. Thus, $\frac{\sigma^{(n)}}{\tau^{(n)}}$ gives the relative temporal position of the first ancestor of the chosen individual/gene among all ancestors of the sample (until the most recent common ancestor). In this sense, we interpret $\frac{\sigma^{(n)}}{\tau^{(n)}}$ as a measure of how ancient the chosen individual/gene is compared to the rest of the sample.

In this article, we focus on the asymptotics of $T^{(n)}, \sigma^{(n)}$ and $R^{(n)}$ for $n \rightarrow \infty$. The asymptotics of these functionals are already known for some $\Lambda$ - $n$-coalescents. For $T^{(n)}$, we have

- $\Lambda=\delta_{0}$ (Kingman's coalescent): $n T^{(n)} \stackrel{d}{\rightarrow} T$, where $T$ has density $t \mapsto \frac{8}{(2+t)^{3}}$ (see [10], [14], [24]),

- $\Lambda=\operatorname{Beta}(1,1)$ (Bolthausen-Sznitman coalescent): $\log (n) T^{(n)} \stackrel{d}{\rightarrow} \operatorname{Exp}(1)$ (see [17]),

- $\Lambda$ with $\mu_{-1}=\int_{0}^{1} x^{-1} \Lambda(d x)<\infty: T^{(n)} \stackrel{d}{\rightarrow} \operatorname{Exp}\left(\mu_{-1}\right)$ (see [29], see also [19])

for $n \rightarrow \infty$. For $\sigma^{(n)}$, we have

- $\Lambda=\delta_{0}: \sigma^{(n)} / n \stackrel{d}{\rightarrow} \operatorname{Beta}(1,2)($ see $[14])$,

- $\Lambda=\operatorname{Beta}(1,1): \frac{\log (n)}{n} \sigma^{(n)} \stackrel{d}{\rightarrow} \operatorname{Beta}(1,1)($ see $[17])$,

- $\Lambda$ with $\mu_{-2}=\int_{0}^{1} x^{-2} \Lambda(d x)<\infty: \sigma^{(n)} \stackrel{d}{\rightarrow} G e o\left(\frac{\mu_{-1}}{\mu_{-2}}\right)$ (see [19])

for $n \rightarrow \infty$, where $\operatorname{Geo}(p)$ is the geometric distribution on $\mathbb{N}$ with parameter $p$.

There are some known results for the asymptotics of the block counting process $R^{(n)}$. Define $R=\left(R_{t}\right)_{t \geq 0}$ by setting $R_{t}:=\left|\Pi_{t}\right|$, the number of blocks of a $\Lambda$-coalescent $\Pi=\left(\Pi_{t}\right)_{t \geq 0}$. Note that $R=\lim _{n \rightarrow \infty} R^{(n)}$, if the block counting process $R^{(n)}$ is defined for the restriction of $\Pi$ to $\{1, \ldots, n\}$ for each $n \in \mathbb{N}$. For the small-time behaviour of the block counting process $R$, the following results are known:

- If $\Lambda(d x)=f(x) d x$, where $f(x) \sim A x^{1-\alpha}$ for $1<\alpha<2$ and $\sim$ means that the ratio of the two sides tends to one as $x \rightarrow 0+$. Then

$$
\lim _{t \rightarrow 0+} t^{1 /(\alpha-1)} R_{t}=\left(\frac{\alpha}{A \Gamma(2-\alpha)}\right)^{1 /(\alpha-1)} \quad \text { a.s. }
$$

(see [3] and [4])

- If $\int_{[\epsilon, 1]} x^{-2} \Lambda(d x)=\epsilon^{-\alpha} L_{\epsilon}$ with $\epsilon \in(0,1)$ and $L_{\epsilon}$ slowly varying as $\epsilon \rightarrow 0$. Denote by $g_{\epsilon}:=L_{\epsilon}^{-1} \epsilon^{\alpha-1}$, then

$$
\lim _{\epsilon \rightarrow 0+} \epsilon R_{g_{\epsilon}}=(\Gamma(2-\alpha))^{1 /(1-\alpha)}, \quad \text { in Probability. }
$$

(see $[6])$.

Notice that both cases are complementary. The block counting process of Kingman coalescent gets similar limit value almost surely by taking $\alpha=2$, (see [3], page 216). 
- For any $\Lambda$ coalescent that comes down from infinity(that means for any $t>0, R_{t}<\infty$ almost surely), there exists a deterministic positive function $v_{t}$ on $(0, \infty)$, such that:

$$
\lim _{t \rightarrow 0+} \frac{R_{t}}{v_{t}}=1, \quad \text { a.s. }
$$

For explicit form of $v$ and finer results, we refer to [2]. This result is much more general than the two former ones.

We will analyze the asymptotics for $T^{(n)}, \sigma^{(n)}$ and $R^{(n)}$ for $\Lambda$-n-coalescents with $\Lambda$ fulfilling

$$
\rho(t)=C_{0} t^{-\alpha}+O\left(t^{-\alpha+\zeta}\right), t \rightarrow 0
$$

for some $C_{0}>0, \alpha \in(1,2)$ and $\zeta>1-1 / \alpha$, where $\rho(t)=\int_{t}^{1} x^{-2} \Lambda(d x)$. Note that this class of $n$-coalescents includes all $\operatorname{Bet} a(a, b)$ - $n$-coalescents with parameters $a \in(0,1)$ and $b>0$. In this class of $n$-coalescents, we have the following asymptotics for $T^{(n)}, \sigma^{(n)}$ and $R^{(n)}$ :

- $\frac{\sigma^{(n)}}{n(\alpha-1)} \stackrel{d}{\rightarrow} \sigma$,

- $n^{\alpha-1} T^{(n)} \stackrel{d}{\rightarrow} \frac{1}{C_{0} \Gamma(2-\alpha)}\left((1-\sigma)^{1-\alpha}-1\right)$,

- for any $t_{0}>0, \varepsilon>0, \mathbb{P}\left(\sup _{0 \leq t \leq t_{0}}\left|n^{-1} R_{t n^{1-\alpha}}^{(n)}-\left(1+C_{0} \Gamma(2-\alpha) t\right)^{-1 /(\alpha-1)}\right|>\varepsilon\right) \rightarrow 0$,

for $n \rightarrow \infty$, where $\sigma \stackrel{d}{=} \operatorname{Beta}(1, \alpha)$.

Remark that if $\Lambda(d x)=f(x) d x$, where $f(x) \sim A x^{1-\alpha}$ for $1<\alpha<2$, we get that for fixed $t>0$,

$$
\lim _{n \rightarrow \infty} \frac{1}{n}\left(1+\frac{A \Gamma(2-\alpha)}{\alpha} t\right)^{1 /(\alpha-1)} R_{t n^{1-\alpha}}^{(n)}=1
$$

in probability, which is to be compared to the following a.s. convergence implied by (2):

$$
\lim _{n \rightarrow \infty} \frac{1}{n}\left(\frac{A \Gamma(2-\alpha)}{\alpha} t\right)^{1 /(\alpha-1)} R_{t n^{1-\alpha}}=1 .
$$

The different scaling comes from the fact that we do not consider the block counting process $R=\lim _{n \rightarrow \infty} R^{(n)}$ of a $\Lambda$-coalescent $\Pi$, but instead the time-changed, scaled limit $\lim _{n \rightarrow \infty} \frac{1}{n} R_{t n^{1-\alpha}}^{(n)}$. To our best knowledge, there does not seem to be a direct link between these two results.

Note that if we see the Bolthausen-Sznitman $n$-coalescent as a Beta $(1,1)$-coalescent and Kingman's $n$-coalescent as the borderline case of a Beta distribution with parameter $\alpha \rightarrow 2$, the convergence results for $\sigma^{(n)}$ shows a nice continuity in the parameters of the limit distributions in the range of $\operatorname{Beta}(2-\alpha, \alpha)$-n-coalescents with $\alpha \in[1,2]$. Our convergence result itself is even somewhat true in the border cases 1 and 2 (if one wages $(\alpha-1)^{-1} \rightarrow \infty$ for $\alpha \rightarrow 1$ against $\log (n) \rightarrow \infty$ for $n \rightarrow \infty$ for the Bolthausen-Sznitman $n$-coalescents). Also note that $T$ obtained as the limit variable of $n T^{(n)}$ in Kingman's case has the same law as $2\left((1-\sigma)^{-1}-1\right)$, which gives again a nice continuity in results. The continuity also appears for the block counting process: replacing $\alpha$ by 2 in the formula for the $\operatorname{Bet} a(2-\alpha, \alpha)-n$-coalescent gives the formula for Kingman's, this result will be proved in the sequel.

Finally we can remark that together with the known asymptotics $\frac{\tau^{(n)}}{n} \stackrel{d}{\rightarrow} \alpha-1$ for this class of $n$-coalescents (see [15], [20] and [23]), we have $\sigma^{(n)} / \tau^{(n)} \stackrel{d}{\rightarrow} \sigma$.

To prove these results, we will exploit some techniques from [15]. In [15], they were used to analyze the asymptotics of a part of the length of a $n$-coalescent and the number of collisions in a $n$-coalescent for the same class of $n$-coalescents as analyzed in the present paper. For the convergence result for $T^{(n)}$, we present two proofs. One mimics the approach 
in [14] and [24], using the convergence result for $\sigma^{(n)}$ and $T^{(n)}=\sum_{i=1}^{\sigma^{(n)}} T_{i}$, where $T_{i}$ is the waiting time between the $(i-1)$ th and $i$ th collision/jump of the $n$-coalescent. The other proof is based on the representation of $T^{(n)}$ as the first jump time of a Cox process driven by a random rate process which depends only on the block counting process associated with the remaining individuals labelled $\{2,3, \ldots, n\}$. We use a recursive construction suitable for any $\Lambda$ - $n$-coalescent: This construction consists in adding individual $i$ to a coalescent process constructed by individuals from 1 to $n$ except $i$ such that consistence relationship is fulfilled.

1.2. Organization of the paper. In section 2, we recall some known technical results which can all be found in [15]. In section 3, we obtain the asymptotic result about $\sigma^{(n)}$ and also about the ratio between $\sigma^{(n)}$ and $\tau^{(n)}$. Section 4 studies the small time behavior of the block counting process $R^{(n)}$. Depending on the property of $R^{(n)}$, our first method taking $T^{(n)}$ as the first jump time of a Cox process gives the asymptotic behavior of $T^{(n)}$ in section 5 . In section 6 , another method is provided by taking into account the fact that $T^{(n)}$ is the sum of $\sigma^{(n)}$ initial waiting times for the coalescent process $\Pi^{(n)}$ to jump from one state to the following.

\section{Preliminaries}

In this Section, we recall some results from [15].

Consider a $n$-coalescent with multiple collisions characterized by a finite measure $\Lambda$ on $[0,1]$. Let $\nu(d x)=x^{-2} \Lambda(d x)$ and $\rho(t)=\nu[t, 1]$. When the process has $k$ blocks, the next coalescence event comes at rate $g_{k}$ given by

$$
g_{k}=\sum_{\ell=1}^{k-1}\left(\begin{array}{c}
k \\
\ell+1
\end{array}\right) \lambda_{k, \ell+1}=\int_{(0,1)}\left(1-(1-x)^{k}-k x(1-x)^{k-1}\right) \frac{\Lambda(d x)}{x^{2}} .
$$

For $n \geq 1, x \in(0,1)$, let $B_{n, x}$ be a binomial r.v. with parameter $(n, x)$. Recall that for $1 \leq k \leq n$, we have

$$
\mathbb{P}\left(B_{n, x} \geq k\right)=\frac{n !}{(k-1) !(n-k) !} \int_{0}^{x} t^{k-1}(1-t)^{n-k} d t .
$$

Use the first equality in (3) and (4) to get

$$
\begin{aligned}
g_{n} & =\int_{0}^{1} \sum_{k=2}^{n}\left(\begin{array}{l}
n \\
k
\end{array}\right) x^{k}(1-x)^{n-k} \nu(d x) \\
& =\int_{0}^{1} \mathbb{P}\left(B_{n, x} \geq 2\right) \nu(d x) \\
& =n(n-1) \int_{0}^{1}(1-t)^{n-2} t \rho(t) d t .
\end{aligned}
$$

All along this paper, the following hypothesis will be assumed

$$
\rho(t)=C_{0} t^{-\alpha}+O\left(t^{-\alpha+\zeta}\right)
$$

for some $C_{0}>0, \alpha \in(1,2)$ and $\zeta>1-1 / \alpha$. Under this hypothesis, Lemma 2.2 of [15] gives us that, for $n \geq 2$,

$$
g_{n}=C_{0} \Gamma(2-\alpha) n^{\alpha}+O\left(n^{\alpha-\min (\zeta, 1)}\right) .
$$


Recall that we call $\tau^{(n)}$ the number of coalescence events until reaching the common ancestor of the initial population (of size $n$ ). For $k \geq 0$, denote by $Y_{k}^{(n)}$ the number of blocks remaining after $k$ jumps. Notice that $Y^{(n)}$ is a decreasing Markov chain with $Y_{0}^{(n)}=n$ and $Y_{k}^{(n)}=1$ for $k \geq \tau^{(n)}$. Let $X_{k}^{(n)}=Y_{k-1}^{(n)}-Y_{k}^{(n)}$ be the number of blocks we lose during the $k$ th coalescence event. We write $X_{0}^{(n)}=0$.

The Markov property makes that the law of the first jump $X_{1}^{(n)}$ will be of much interest. We will look at some properties of $X_{1}^{(n)}$. Notice that

$$
\mathbb{P}\left(X_{1}^{(n)}=k\right)=\frac{1}{g_{n}} \int_{0}^{1} \mathbb{P}\left(B_{n, x}=k+1\right) \nu(d x)
$$

and thus

$$
\mathbb{P}\left(X_{1}^{(n)} \geq k\right)=\frac{\int_{0}^{1} \mathbb{P}\left(B_{n, x} \geq k+1\right) \nu(d x)}{g_{n}}=\frac{(n-2) !}{k !(n-k-1) !} \frac{\int_{0}^{1}(1-t)^{n-k-1} t^{k} \rho(t) d t}{\int_{0}^{1}(1-t)^{n-2} t \rho(t) d t} .
$$

Under the same assumptions on $\rho(t)$, setting $\varepsilon_{0}>0$ and

$$
\varphi_{n}= \begin{cases}n^{-\zeta} & \text { if } \quad \zeta<\alpha-1 \\ n^{1-\alpha+\varepsilon_{0}} & \text { if } \quad \zeta=\alpha-1 \\ n^{1-\alpha} & \text { if } \quad \zeta>\alpha-1\end{cases}
$$

Lemma 2.3 of [15] tells us there exists a constant $C_{10}$ s.t. for all $n \geq 2$, we have

$$
\left|\mathbb{E}\left[X_{1}^{(n)}\right]-\frac{1}{\alpha-1}\right| \leq C_{10} \varphi_{n}
$$

Moreover, from Lemma 2.4 of [15], there exists a constant $C_{11}$ s.t. for all $n \geq 2$, we have

$$
\mathbb{E}\left[\left(X_{1}^{(n)}\right)^{2}\right] \leq C_{11} \frac{n^{2}}{g_{n}}
$$

We consider $\phi_{n}$ the Laplace transform of $X_{1}^{(n)}$ : for $u \geq 0, \phi_{n}(u)=\mathbb{E}\left[\mathrm{e}^{-u X_{1}^{(n)}}\right]$. Assume that hypothesis (5) holds true. Let $\varepsilon_{0}>0$. Recall $\varphi_{n}$ given by (9). Then we have (see [15], Lemma 2.5), for $n \geq 2$,

$$
\phi_{n}(u)=1-\frac{u}{\alpha-1}+\frac{u^{\alpha}}{\alpha-1}+R(n, u),
$$

where $R(n, u)=\left(u \varphi_{n}+u^{2}\right) h(n, u)$ with $\sup _{u \in[0, K], n \geq 2}|h(n, u)|<\infty$ for all $K>0$.

Moreover, if we assume that $\zeta>1-1 / \alpha$ and set $\eta \geq \frac{1}{\alpha}$, then (from [15], Lemma 3.2) there exist $\varepsilon_{1}>0$ and $C_{13}(K)$ a finite constant such that for all $n \geq 1$ and $u \in[0, K]$, a.s. with $a_{n}=n^{-\eta}$,

$$
\sum_{i=1}^{\tau_{n}}\left|R\left(Y_{i-1}^{(n)}, u a_{n}\right)\right| \leq C_{13}(K) n^{-\varepsilon_{1}} .
$$

We will also use the following result : Let $V=\left(V_{t}, t \geq 0\right)$ be a $\alpha$-stable Lévy process with no positive jumps (see chap. VII in [5]) with Laplace exponent $\psi(u)=u^{\alpha} /(\alpha-1)$ : for all $u \geq 0, \mathbb{E}\left[\mathrm{e}^{-u V_{t}}\right]=\mathrm{e}^{t u^{\alpha} /(\alpha-1)}$. We assume that $\rho(t)=C_{0} t^{-\alpha}+O\left(t^{-\alpha+\zeta}\right)$ for some $C_{0}>0$ and 
$\zeta>1-1 / \alpha$. Recall that $\tau^{(n)}$ is the number of coalescing events in the $n$-coalescent until reaching its absorbing state. Let

$$
V_{t}^{(n)}=n^{-1 / \alpha} \sum_{k=1}^{\lfloor n t\rfloor \wedge \tau^{(n)}}\left(X_{k}^{(n)}-\frac{1}{\alpha-1}\right)
$$

for $t \in[0, \alpha-1)$, and

$$
V_{\alpha-1}^{(n)}=n^{-1 / \alpha} \sum_{k=1}^{\tau^{(n)}}\left(X_{k}^{(n)}-\frac{1}{\alpha-1}\right)=n^{-1 / \alpha}\left(n-1-\frac{\tau^{(n)}}{\alpha-1}\right) .
$$

Then,

$$
\left(V_{t}^{(n)}, t \in[0, \alpha-1]\right) \rightarrow\left(V_{t}, t \in[0, \alpha-1]\right)
$$

in the sense of convergence in law of the finite-dimensional marginals (see [15], Corollary 3.5, see also $[20,23])$.

\section{THE NUMBER OF COLLISIONS IN AN EXTERNAL BRANCH}

Consider a $n$-coalescent with multiple collisions characterized by a finite measure $\Lambda$ on $[0,1]$. Recall that $\nu(d x)=x^{-2} \Lambda(d x)$ and $\rho(t)=\nu[t, 1]$ which is assumed to satisfy (5). A $n$-coalescent takes its values in $\mathcal{P}^{(n)}$, the set of partitions of $\{1, \ldots, n\}$. For $i \geq 0$, let $\pi_{i}=\pi_{i}^{(n)}$ be the state of the process after the $i$ th coalescence event.

Pick at random an individual from the initial population and denote by $T^{(n)}$ the length of the external branch starting from it. Because of exchangeability, $T^{(n)}$ has the same law as the length $T_{1}^{(n)}$ of the external branch starting from the initial individual labelled by $\{1\}$. A quantity of interest will be $\sigma^{(n)}$, the number of coalescence events we have to wait to see the randomly chosen external branch merging. Again because of exchangeability, $\sigma^{(n)}$ has the same law as

$$
\sigma_{1}^{(n)}=\inf \left\{i>0,\{1\} \notin \pi_{i}\right\},
$$

the time we have to wait to see the external branch linked to individual 1 merging. We can write

$$
T_{1}^{(n)}=\sum_{i=1}^{\sigma_{1}^{(n)}} \frac{e_{i}}{g_{Y_{i-1}^{(n)}}}
$$

where the $e_{i}$ 's are i.i.d. exponential random variables with mean 1 . Note that the formula also holds true for $T^{(n)}$ and $\sigma^{(n)}$ (just omit the subscripts). For the remainder of the chapter, we will identify $\sigma^{(n)}$ with $\sigma_{1}^{(n)}$.

In this section, we will determinate the asymptotic law of $\sigma^{(n)}$ for a class of coalescents containing the Beta-coalescent with $\alpha \in(1,2)$.

Theorem 3.1. We assume that $\rho(t)=C_{0} t^{-\alpha}+O\left(t^{-\alpha+\zeta}\right)$ for some $C_{0}>0, \alpha \in(1,2)$ and $\zeta>1-1 / \alpha$. Then

$$
\frac{\sigma^{(n)}}{n(\alpha-1)} \stackrel{d}{\rightarrow} \sigma
$$

for $n \rightarrow \infty$, where $\sigma \stackrel{d}{=} \operatorname{Beta}(1, \alpha)$. 
Recall that in this class of $n$-coalescents, we also have $\tau^{(n)} / n \stackrel{d}{\rightarrow} \alpha-1$ (from [15], see also [20] and [23]) for $n \rightarrow \infty$. Slutsky's theorem gives a convergence result for $\sigma^{(n)} / \tau^{(n)}$, which measures how ancient the chosen individual is compared to the rest of the sample

Corollary 3.2. We assume that $\rho(t)=C_{0} t^{-\alpha}+O\left(t^{-\alpha+\zeta}\right)$ for some $C_{0}>0, \alpha \in(1,2)$ and $\zeta>1-1 / \alpha$. Then

$$
\frac{\sigma^{(n)}}{\tau^{(n)}} \stackrel{d}{\rightarrow} \sigma
$$

for $n \rightarrow \infty$, where $\sigma \stackrel{d}{=} \operatorname{Beta}(1, \alpha)$.

proof of Theorem 3.1. For convenience, we set

(1) $\left(\begin{array}{l}a \\ b\end{array}\right)=0$, if $0 \leq a<b, a \in \mathbb{Z}_{+}, b \in \mathbb{Z}_{+}, \mathbb{Z}_{+}=\{0,1,2, \ldots\}$.

(2) $\log (0)=-\infty$.

Notice that $\sigma^{(n)} \leq \tau^{(n)}$. Let $\mathcal{Y}=\left(\mathcal{Y}_{k}, k \geq 0\right)$ denotes the filtration generated by $Y^{(n)}$. For any $t \geq 0$, we have

$$
\begin{aligned}
\mathbb{P}\left(\sigma^{(n)}>n t \mid \mathcal{Y}\right) & =\mathbb{P}\left(\sigma^{(n)}>n t, \tau^{(n)}>n t \mid \mathcal{Y}\right) \\
& =\prod_{i=1}^{\lfloor n t\rfloor \wedge \tau^{(n)}} \mathbb{P}\left(\{1\} \in \pi_{i} \mid\{1\} \in \pi_{i-1}, \mathcal{Y}\right) \\
& =\prod_{i=1}^{\lfloor n t\rfloor \wedge \tau^{(n)}} \frac{\left(\begin{array}{l}
Y_{i-1}^{(n)}-1 \\
X_{i}^{(n)}+1
\end{array}\right)}{\left(\begin{array}{c}
Y_{i-1}^{(n)} \\
X_{i}^{(n)}+1
\end{array}\right)} \\
& =\prod_{i=1}^{\lfloor n t\rfloor \wedge \tau^{(n)}} \frac{Y_{i-1}^{(n)}-\left(X_{i}^{(n)}+1\right)}{Y_{i-1}^{(n)}} .
\end{aligned}
$$

Notice that $\frac{X_{i}^{(n)}+1}{Y_{i-1}^{(n)}}<1$ if $i \neq \tau^{(n)}$, and $\frac{X_{i}^{(n)}+1}{Y_{i-1}^{(n)}}=1$ if $i=\tau^{(n)}$.

We can hence write

$$
\log \left(\mathbb{P}\left(\sigma^{(n)}>n t \mid \mathcal{Y}\right)\right)=\sum_{i=1}^{\lfloor n t\rfloor \wedge \tau^{(n)}} \log \left(1-\frac{X_{i}^{(n)}+1}{Y_{i-1}^{(n)}}\right)
$$

and proceed to a power series expansion :

$$
\log \left(\mathbb{P}\left(\sigma^{(n)}>n t \mid \mathcal{Y}\right)\right)=I_{n t}^{(1)}+I_{n t}^{(2)}
$$

with

$$
I_{n t}^{(1)}=-\sum_{i=1}^{\lfloor n t\rfloor \wedge \tau^{(n)}}\left(X_{i}^{(n)}+1\right)\left(Y_{i-1}^{(n)}\right)^{-1}
$$


and

$$
I_{n t}^{(2)}=\sum_{i=1}^{\lfloor n t\rfloor \wedge \tau^{(n)}}\left(\log \left(1-\frac{X_{i}^{(n)}+1}{Y_{i-1}^{(n)}}\right)+\frac{X_{i}^{(n)}+1}{Y_{i-1}^{(n)}}\right),
$$

where $I_{n t}^{(2)}$ can be $-\infty$ if $\frac{X_{i}^{(n)}+1}{Y_{i-1}^{(n)}}=1$. Let us look further at $I_{n t}^{(1)}$. The idea is to replace $X_{i}^{(n)}$ by the limit of its expectation.

$$
I_{n t}^{(1)}=J_{n t}^{(1)}+J_{n t}^{(2)}
$$

with

$$
J_{n t}^{(1)}=-\sum_{i=1}^{\lfloor n t\rfloor \wedge \tau^{(n)}}\left(\frac{1}{\alpha-1}+1\right)\left(Y_{i-1}^{(n)}\right)^{-1}
$$

and

$$
J_{n t}^{(2)}=-\sum_{i=1}^{\lfloor n t\rfloor \wedge \tau^{(n)}}\left(X_{i}^{(n)}-\frac{1}{\alpha-1}\right)\left(Y_{i-1}^{(n)}\right)^{-1} .
$$

We will use three lemmas whose proofs are given in the rest of the Section.

Lemma 3.3, with $\eta=1$, tells us that, when $0<t<\alpha-1$

$$
J_{n t}^{(1)} \stackrel{\mathbb{P}}{\rightarrow}-\frac{\alpha}{\alpha-1} \int_{0}^{t}\left(1-\frac{x}{\alpha-1}\right)^{-1} d x=\alpha \log \left(1-\frac{t}{\alpha-1}\right) .
$$

Lemma 3.4 gives, for $0<t<\alpha-1$,

$$
J_{n t}^{(2)}=-n^{1 / \alpha-1} n^{1-1 / \alpha} \sum_{i=1}^{\lfloor n t\rfloor \wedge \tau^{(n)}}\left(X_{i}^{(n)}-\frac{1}{\alpha-1}\right)\left(Y_{i-1}^{(n)}\right)^{-1} \stackrel{\mathbb{P}}{\rightarrow} 0 .
$$

Finally, Lemma 3.5 gives, for $t<\alpha-1$,

$$
I_{n t}^{(2)} \stackrel{\mathbb{P}}{\rightarrow} 0 .
$$

Adding (17), (18) and (19), we get that for $t<\alpha-1$

$$
\log \left(\mathbb{P}\left(\sigma^{(n)}>n t \mid \mathcal{Y}\right)\right) \stackrel{\mathbb{P}}{\rightarrow} \alpha \log \left(1-\frac{t}{\alpha-1}\right),
$$

and thus

$$
\mathbb{P}\left(\sigma^{(n)}>n t \mid \mathcal{Y}\right) \stackrel{\mathbb{P}}{\rightarrow}\left(1-\frac{t}{\alpha-1}\right)^{\alpha} .
$$

While we know that $\mathbb{P}\left(\sigma^{(n)}>n t \mid \mathcal{Y}\right) \leq 1$, then

$$
\mathbb{P}\left(\sigma^{(n)}>n t\right)=\mathbb{E}\left[\mathbb{P}\left(\sigma^{(n)}>n t \mid \mathcal{Y}\right)\right] \rightarrow\left(1-\frac{t}{\alpha-1}\right)^{\alpha} .
$$

We thus obtain that, for $x \in(0,1)$,

$$
\mathbb{P}\left(\sigma^{(n)}>n(\alpha-1) x\right) \rightarrow(1-x)^{\alpha} .
$$

and then that $\frac{\sigma^{(n)}}{n(\alpha-1)}$ converges in distribution to a $\operatorname{Beta}(1, \alpha)$ law. 
Lemma 3.3. We set $\nu_{\eta}(t)=\int_{0}^{t}\left(1-\frac{x}{\alpha-1}\right)^{-\eta} d x, \eta \in \mathbb{R}$. We assume that $\rho(t)=C_{0} t^{-\alpha}+$ $O\left(t^{-\alpha+\zeta}\right)$ for some $C_{0}>0, \alpha \in(1,2)$ and $\zeta>1-1 / \alpha$. For any $0<t<\alpha-1$ and $\eta \in \mathbb{R}$, we have

(1) Let $t_{0} \in[0, \alpha-1)$ and $\delta>0$. The following convergence in probability holds when $n \rightarrow \infty$ :

$$
n^{(\alpha-1) / 2-\delta} \sup _{0 \leq t \leq t_{0}}\left|n^{\eta-1} \sum_{i=1}^{\lfloor n t\rfloor \wedge \tau^{(n)}}\left(Y_{i-1}^{(n)}\right)^{-\eta}-\nu_{\eta}(t)\right| \rightarrow 0 .
$$

(2) Let $t \in[0, \alpha-1)$. The following convergence in distribution holds when $n \rightarrow \infty$ :

$$
n^{\eta-1 / \alpha}\left(\sum_{i=1}^{\lfloor n t\rfloor \wedge \tau^{(n)}}\left(Y_{i-1}^{(n)}\right)^{-\eta}-n^{1-\eta} \nu_{\eta}(t)\right) \rightarrow \eta \int_{0}^{t} d r\left(1-\frac{r}{\gamma}\right)^{-\eta-1} V_{r}
$$

Proof. The case $\eta=\alpha-1$ is given by Theorem 5.1 in [15]. Following the same arguments, it is easy to get the general result.

Lemma 3.4. For any $t<\alpha-1$, the following convergence in distribution holds :

$$
n^{1-1 / \alpha} \sum_{i=1}^{\lfloor n t\rfloor \wedge \tau^{(n)}}\left(X_{i}^{(n)}-\frac{1}{\alpha-1}\right)\left(Y_{i-1}^{(n)}\right)^{-1} \stackrel{d}{\rightarrow}\left(v_{\alpha}(t)\right)^{1 / \alpha} V_{1},
$$

where $\left(V_{t}\right)_{t \geq 0}$ is an $\alpha$-stable Lévy process with no positive jumps and $v_{\alpha}(t)=\int_{0}^{t}\left(1-\frac{x}{\alpha-1}\right)^{-\alpha} d x$.

Proof. Let $\delta \in(0, \alpha-1), t_{0}=\alpha-1-\delta$ and $t \in\left[0, t_{0}\right]$.

Let $\varepsilon \in\left(0,1-\frac{t}{\alpha-1}\right)$ and $\beta=1-\frac{t}{\alpha-1}-\varepsilon>0$. We have

$$
n^{1-1 / \alpha} \sum_{i=1}^{\lfloor n t\rfloor \wedge \tau^{(n)}}\left(X_{i}^{(n)}-\frac{1}{\alpha-1}\right)\left(Y_{i-1}^{(n)}\right)^{-1}=A_{n t}+B_{n t},
$$

with

$$
A_{n t}=n^{1-1 / \alpha} \sum_{i=1}^{\lfloor n t\rfloor \wedge \tau^{(n)}}\left(X_{i}^{(n)}-\frac{1}{\alpha-1}\right)\left(Y_{i-1}^{(n)}\right)^{-1} \mathbf{1}_{\left\{Y_{i-1}^{(n)} \geq n \beta\right\}}
$$

and

$$
B_{n t}=n^{1-1 / \alpha} \sum_{i=1}^{\lfloor n t\rfloor \wedge \tau^{(n)}}\left(X_{i}^{(n)}-\frac{1}{\alpha-1}\right)\left(Y_{i-1}^{(n)}\right)^{-1} \mathbf{1}_{\left\{Y_{i-1}^{(n)}<n \beta\right\}} .
$$

We will show that $B_{n t}$ converges to 0 in probability and that $A_{n t}$ weakly converges to $\left(v_{\alpha}(t)\right)^{1 / \alpha} V_{1}$ as $n \rightarrow \infty$.

Convergence of $A_{n t}$. Let $Z_{i}^{(n)}=n\left(Y_{i-1}^{(n)}\right)^{-1} \mathbf{1}_{\left\{Y_{i-1}^{(n)} \geq n \beta\right\}}$. We have that $\sup _{n, i \geq 1} Z_{i}^{(n)} \leq \beta^{-1}$ a.s..

By using (12), it is enough to prove that

$$
\mathbb{E}\left[\exp \left(-u A_{n t}\right)\right] \underset{n \rightarrow \infty}{\longrightarrow} \mathrm{e}^{v_{\alpha}(t) u^{\alpha} /(\alpha-1)}
$$

for any $u$ positive, where $\mathrm{e}^{v_{\alpha}(t) u^{\alpha} /(\alpha-1)}$ is the Laplace transform of $\left(v_{\alpha}(t)\right)^{1 / \alpha} V_{1}$.

Taking $u Z_{i}^{(n)}$ as $Z_{i}^{(n)}$, we shall only consider the case $u=1$. 
Let us consider $n^{-1} \sum_{i=1}^{\lfloor n t\rfloor \wedge \tau^{(n)}}\left(Z_{i}^{(n)}\right)^{\alpha}=n^{\alpha-1} \sum_{i=1}^{\lfloor n t\rfloor \wedge \tau^{(n)}}\left(Y_{i-1}^{(n)}\right)^{-\alpha} \mathbf{1}_{\left\{Y_{i-1}^{(n)} \geq n \beta\right\}}$. We have, because the process $\left(Y_{i}^{(n)}, i \geq 0\right)$ is decreasing, that

$$
\begin{aligned}
\mathbb{P}\left(n^{-1} \sum_{i=1}^{\lfloor n t\rfloor \wedge \tau^{(n)}}\left(Z_{i}^{(n)}\right)^{\alpha} \neq n^{\alpha-1} \sum_{i=1}^{\lfloor n t\rfloor \wedge \tau^{(n)}}\left(Y_{i-1}^{(n)}\right)^{-\alpha}\right) & =\mathbb{P}\left(\exists i ; Y_{i-1}^{(n)}<n \beta\right) \\
& \leq \mathbb{P}\left(\left\{Y_{\left(\lfloor n t\rfloor \wedge \tau^{(n)}\right)-1}^{(n)}<n \beta\right)\right. \\
& \leq \mathbb{P}\left(n^{-1} \sum_{j=1}^{\left(\lfloor n t\rfloor \wedge \tau^{(n)}\right)-1}\left(X_{j}^{(n)}-\frac{1}{\alpha-1}\right) \geq \varepsilon\right) .
\end{aligned}
$$

Use (14) to get that the right-hand side of the last inequality converges to 0 as $n$ goes to infinity. Using also Lemma 3.3 with $\eta=\alpha$, we have that

$$
n^{\alpha-1} \sum_{i=1}^{\lfloor n t\rfloor \wedge \tau^{(n)}}\left(Y_{i-1}^{(n)}\right)^{-\alpha} \stackrel{\mathbb{P}}{\rightarrow} v_{\alpha}(t)
$$

as $n \rightarrow \infty$. We can thus deduce that

$$
n^{-1} \sum_{i=1}^{\lfloor n t\rfloor \wedge \tau^{(n)}}\left(Z_{i}^{(n)}\right)^{\alpha} \stackrel{\mathbb{P}}{\rightarrow} v_{\alpha}(t),
$$

as $n \rightarrow \infty$.

For $a>0$, we set

$$
M_{n, k}^{(a)}=\exp \left(\sum_{i=1}^{k}\left(-n^{-1 / \alpha} a Z_{i}^{(n)} X_{i}^{(n)}-\log \phi_{Y_{i-1}^{(n)}}\left(n^{-1 / \alpha} a Z_{i}^{(n)}\right)\right)\right) .
$$

The process $\left(M_{n, k}^{(a)}, k \geq 1\right)$ is a bounded martingale w.r.t. the filtration $\mathcal{Y}$. Notice that $\mathbb{E}\left[M_{n, k}^{(a)}\right]=1$. As $X_{i}^{n}=0$ and $Z_{i}^{(n)}=0$ for $i>\tau^{(n)}$, we also have

$$
M_{n, k}^{(a)}=\exp \left(\sum_{i=1}^{k \wedge \tau^{(n)}}\left(-n^{-1 / \alpha} a Z_{i}^{(n)} X_{i}^{(n)}-\log \phi_{Y_{i-1}^{(n)}}\left(n^{-1 / \alpha} a Z_{i}^{(n)}\right)\right)\right) .
$$

Using $R(n, u)$ defined in (12), we get that :

$$
\begin{aligned}
& M_{n,\lfloor n t\rfloor}^{(a)} \\
& =\exp \left(-\sum_{i=1}^{\lfloor n t\rfloor \wedge \tau^{(n)}} n^{-1 / \alpha} a Z_{i}^{(n)}\left(X_{i}^{(n)}-\frac{1}{\alpha-1}\right)-\sum_{i=1}^{\lfloor n t\rfloor \tau^{(n)}} \frac{n^{-1}\left(a Z_{i}^{(n)}\right)^{\alpha}}{\alpha-1}-\sum_{i=1}^{\lfloor n t\rfloor \wedge \tau^{(n)}} R\left(Y_{k-1}^{(n)}, n^{-1 / \alpha} a Z_{i}^{(n)}\right)\right) \\
& =\exp \left(-a A_{n t}\right) \exp \left(-n^{-1} \sum_{i=1}^{\lfloor n t\rfloor \wedge \tau^{(n)}} \frac{\left(a Z_{i}^{(n)}\right)^{\alpha}}{\alpha-1}-\sum_{i=1}^{\lfloor n t\rfloor \wedge \tau^{(n)}} R\left(Y_{k-1}^{(n)}, n^{-1 / \alpha} a Z_{i}^{(n)}\right)\right) .
\end{aligned}
$$

Let

$$
\Lambda_{n}=-n^{-1} \sum_{i=1}^{\lfloor n t\rfloor \wedge \tau^{(n)}} \frac{\left(Z_{i}^{(n)}\right)^{\alpha}}{\alpha-1}+\frac{v_{\alpha}(t)}{\alpha-1}
$$


and write

$$
\mathbb{E}\left[\exp \left(-A_{n t}\right)\right]=A_{1}+A_{2}
$$

with $A_{1}=\mathbb{E}\left[\mathrm{e}^{-A_{n t}}\left(1-\mathrm{e}^{\Lambda_{n}}\right)\right]$ and $A_{2}=\mathbb{E}\left[\mathrm{e}^{-A_{n t}} \mathrm{e}^{\Lambda_{n}}\right]$

First of all, let us prove that $A_{1}$ converges to 0 when $n$ tends to $\infty$. Recall that the r.v $Z_{i}^{(n)}$ are uniformly bounded by $\beta^{-1}$ a.s.. Thanks to (13), we have

$\mathbb{E}\left[\mathrm{e}^{-2 A_{n t}}\right]=\mathbb{E}\left[M_{n,\lfloor n t\rfloor}^{(2)} \exp \left(n^{-1} \sum_{i=1}^{\lfloor n t\rfloor \wedge \tau^{(n)}} \frac{\left(2 Z_{i}^{(n)}\right)^{\alpha}}{\alpha-1}+\sum_{i=1}^{\lfloor n t\rfloor \wedge \tau^{(n)}} R\left(Y_{k-1}^{(n)}, 2 n^{-1 / \alpha} Z_{i}^{(n)}\right)\right)\right] \leq M$,

where $M$ is a finite constant which does not depend on $n$. By Cauchy-Schwarz' inequality, we get that

$$
\left(A_{1}\right)^{2} \leq\left(\mathbb{E}\left[\mathrm{e}^{-A_{n t}}\left|1-\mathrm{e}^{\Lambda_{n}}\right|\right]\right)^{2} \leq \mathbb{E}\left[\mathrm{e}^{-2 A_{n t}}\right] \mathbb{E}\left[\left(1-\mathrm{e}^{\Lambda_{n}}\right)^{2}\right] \leq M \mathbb{E}\left[\left(1-\mathrm{e}^{\Lambda_{n}}\right)^{2}\right]
$$

The quantity $\Lambda_{n}$ is bounded and goes to 0 in probability when $n$ goes to infinity (see (20)). Therefore, the right-hand side of (21) converges to 0 . This implies that $\lim _{n \rightarrow \infty} A_{1}=0$.

Let us now consider the convergence of $A_{2}$. Remark that

$$
A_{2}=\mathbb{E}\left[M_{n,\lfloor n t\rfloor}^{(1)} \exp \left(\frac{v_{\alpha}(t)}{\alpha-1}+\sum_{k=1}^{\lfloor n t\rfloor \wedge \tau^{(n)}} R\left(Y_{k-1}^{(n)}, n^{-1 / \alpha} Z_{i}^{(n)}\right)\right)\right] .
$$

Recall that $\mathbb{E}\left[M_{n,\lfloor n t\rfloor}^{(1)}\right]=1$. Using (13), we get

$$
\exp \left(-C_{13}\left(\beta^{-1}\right) n^{-\varepsilon_{1}}+\frac{v_{\alpha}(t)}{\alpha-1}\right) \leq A_{2} \leq \exp \left(C_{13}\left(\beta^{-1}\right) n^{-\varepsilon_{1}}+\frac{v_{\alpha}(t)}{\alpha-1}\right)
$$

We get that $\lim _{n \rightarrow \infty} A_{2}=\mathrm{e}^{v_{\alpha}(t) /(\alpha-1)}$, which achieves the proof.

Convergence of $B_{n t}$. Here, we will use a similar approach as the one we used on the first half of p.11. The process $\left(Y_{i}^{(n)}, i \geq 0\right)$ is decreasing. So if for some $i \leq\lfloor n t\rfloor, Y_{i-1}^{(n)}<n \beta$, then we have $Y_{\lfloor n t\rfloor-1}^{(n)}<n \beta$. Thus we get $B_{n t}=B_{n t} \mathbf{1}_{\left\{Y_{(\lfloor n t\rfloor \wedge \tau}^{(n)}(n)-1\right.}^{<n \beta\}}$. Moreover,

$$
\left\{Y_{\left(\lfloor n t\rfloor \wedge \tau^{(n)}\right)-1}^{(n)}<n \beta\right\} \subset\left\{n^{-1} \sum_{j=1}^{\left(\lfloor n t\rfloor \wedge \tau^{(n)}\right)-1}\left(X_{j}^{(n)}-\frac{1}{\alpha-1}\right) \geq \varepsilon\right\}
$$

and then for any $\varepsilon^{\prime}>0$

$$
\begin{aligned}
\mathbb{P}\left(\left|B_{n t}\right| \geq \varepsilon^{\prime}\right) & =\mathbb{P}\left(\mathbf{1}_{\left\{Y_{\left(\lfloor n t\rfloor \wedge \tau^{(n)}\right)-1}^{(n)}<n \beta\right\}}\left|B_{n t}\right| \geq \varepsilon^{\prime}\right) \\
& \leq \mathbb{P}\left(\left\{Y_{\left(\lfloor n t\rfloor \wedge \tau^{(n)}\right)-1}^{(n)}<n \beta\right)\right. \\
& \leq \mathbb{P}\left(n^{-1} \sum_{j=1}^{\left(\lfloor n t\rfloor \wedge \tau^{(n)}\right)-1}\left(X_{j}^{(n)}-\frac{1}{\alpha-1}\right) \geq \varepsilon\right) .
\end{aligned}
$$

Use (14) to get that the right-hand side of the last inequality converges to 0 as $n$ goes to infinity.

Now we deal with $I_{n t}^{(2)}$. 
Lemma 3.5. We assume that $\rho(t)=C_{0} t^{-\alpha}+O\left(t^{-\alpha+\zeta}\right)$ for some $C_{0}>0$ and $\zeta>1-1 / \alpha$. Then, for any $t<\alpha-1$, we have

$$
\left|I_{n t}^{(2)}\right|=\sum_{i=1}^{\lfloor n t\rfloor \wedge \tau^{(n)}}-\left(\log \left(1-\frac{X_{i}^{(n)}+1}{Y_{i-1}^{(n)}}\right)+\frac{X_{i}^{(n)}+1}{Y_{i-1}^{(n)}}\right) \stackrel{\mathbb{P}}{\rightarrow} 0,
$$

when $n \rightarrow \infty$.

Proof. Let $0 \leq t<\alpha-1$. First of all, remark that:

$$
\frac{Y_{\lfloor n t\rfloor}^{(n)}}{n} \stackrel{\mathbb{P}}{\rightarrow} \frac{\alpha-1-t}{\alpha-1} .
$$

Indeed,

$$
\frac{Y_{\lfloor n t\rfloor}^{(n)}}{n}=\frac{n-(\lfloor n t\rfloor) /(\alpha-1)}{n}-\frac{\sum_{k=1}^{\lfloor n t\rfloor}\left(X_{k}^{(n)}-1 /(\alpha-1)\right)}{n},
$$

and we conclude using (14) and the convergence of $\mathbb{P}\left(\tau^{(n)}>\lfloor n t\rfloor\right)$ to 1 . Let us write

$$
\left|I_{n t}^{(2)}\right|=A_{n}+B_{n},
$$

with

$$
A_{n}=\left|I_{n t}^{(2)}\right| \mathbf{1}_{\left\{Y_{\lfloor n t\rfloor}^{(n)}<(1-t /(\alpha-1)) n / 2\right\}}
$$

and

$$
B_{n}=\left|I_{n t}^{(2)}\right| \mathbf{1}_{\left\{Y_{\lfloor n t\rfloor}^{(n)} \geq(1-t /(\alpha-1)) n / 2\right\}} .
$$

The convergence (22) implies that $A_{n}$ tends to 0 in probability. To prove the convergence of $B_{n}$, let us first notice that for $a \in(0,1)$, there exists a constant $C(a)$ such that, if $B_{n, x}$ is a binomial r.v. with parameter $(n, x)$, then

$$
0<-\int_{0}^{1} \mathbb{E}\left[\mathbf{1}_{\left\{2 \leq B_{n, x} \leq(1-a) n\right\}}\left(\log \left(1-\frac{B_{n, x}}{n}\right)+\frac{B_{n, x}}{n}\right)\right] \nu(d x) \leq C(a) .
$$

Indeed, there exists a constant $C^{\prime}(a)$ such that for $u \in(0,1-a), 0<-\ln (1-u)-u \leq C^{\prime}(a) u^{2}$. Hence,

$$
\begin{aligned}
0 & <-\int_{0}^{1} \mathbb{E}\left[\mathbf{1}_{\left\{2 \leq B_{n, x} \leq(1-a) n\right\}}\left(\log \left(1-\frac{B_{n, x}}{n}\right)+\frac{B_{n, x}}{n}\right)\right] \nu(d x) \\
& \leq C^{\prime}(a) \int_{0}^{1} \mathbb{E}\left[\mathbf{1}_{\left\{2 \leq B_{n, x} \leq(1-a) n\right\}}\left(\frac{B_{n, x}}{n}\right)^{2}\right] \nu(d x) \\
& \leq C^{\prime}(a) \int_{0}^{1} \mathbb{E}\left[\left(\frac{B_{n, x}}{n}\right)^{2}\right] \nu(d x) \\
& \leq 2 C^{\prime}(a) \int_{0}^{1} \mathbb{E}\left[\frac{B_{n, x}\left(B_{n, x}-1\right)}{n^{2}}\right] \nu(d x) \\
& =2 C^{\prime}(a) \frac{\int_{0}^{1} n(n-1) x^{2} \nu(d x)}{n^{2}} \\
& \leq 2 C^{\prime}(a) \Lambda([0,1])=: C(a) .
\end{aligned}
$$


Let us set $a=(1-t /(\alpha-1)) / 2$. Hence $B_{n}=\left|I_{n t}^{(2)}\right| \mathbf{1}_{\left\{Y_{\lfloor n t\rfloor}^{(n)} \geq a n\right\}}$. Notice that if $n$ is large enough such that $a n \geq 2$, then if $Y_{\lfloor n t\rfloor}^{(n)} \geq a n$ we have $\tau^{(n)}>n t$. Moreover, if $Y_{\lfloor n t\rfloor}^{(n)} \geq a n$, for $i \leq n t$, we have $Y_{i}^{(n)} \geq a n \geq a Y_{i-1}^{(n)}$ and $X_{i}^{(n)}=Y_{i-1}^{(n)}-Y_{i}^{(n)} \leq(1-a) Y_{i-1}^{(n)}<(1-a / 2) Y_{i-1}^{(n)}$. Using (7), (23) and (6), we get that

$$
\begin{aligned}
& \mathbb{E}\left[B_{n}\right] \\
\leq & \sum_{i=1}^{\lfloor n t\rfloor} \mathbb{E}\left[\mathbb{E}\left[-\left(\log \left(1-\frac{X_{i}^{(n)}+1}{Y_{i-1}^{(n)}}\right)+\frac{X_{i}^{(n)}+1}{Y_{i-1}^{(n)}}\right) \mathbf{1}_{\left\{1 \leq X_{i}^{(n)} \leq(1-a) Y_{i-1}^{(n)}\right\}} \mathbf{1}_{\left\{Y_{i-1}^{(n)} \geq a n\right\}} \mid Y_{i-1}^{(n)}\right]\right] \\
\leq & \sum_{i=1}^{\lfloor n t\rfloor} \mathbb{E}\left[-\mathbb{E}\left[\int_{0}^{1} \mathbf{1}_{\left\{2 \leq B_{Y_{i-1}^{(n)}, x} \leq(1-a / 2) Y_{i-1}^{(n)}\right\}} \mathbf{1}_{\left\{Y_{i-1}^{(n)} \geq a n\right\}} \frac{1}{g_{Y_{i-1}^{(n)}}}\left(\log \left(1-\frac{B_{Y_{i-1}^{(n)}, x}}{Y_{i-1}^{(n)}}\right)+\frac{B_{Y_{i-1}^{(n)}, x}}{Y_{i-1}^{(n)}}\right) \nu(d x) \mid Y_{i-1}^{(n)}\right]\right] \\
\leq & \frac{C(a / 2) n t}{g_{a n}} \rightarrow 0,
\end{aligned}
$$

when $n$ tends to $\infty$. This achieves the proof of the Lemma.

\section{A Result on SMall-time Behavior of the Block process}

We now turn to the study of the length of an external branch picked at random, denoted by $T^{(n)}$. For any integer $k$ between 1 and $\tau^{(n)}$, define $A_{k}^{(n)}$ as the time when the $k$ th jump is achieved. This variable can be expressed as a sum of $k$ independent exponential random variables. More precisely,

$$
A_{k}^{(n)}=\sum_{i=1}^{k \wedge \tau^{(n)}} \frac{e_{i}}{g_{Y_{i-1}^{(n)}}}
$$

where the $e_{i}$ 's are independent standard exponential variables. Notice that $T^{(n)}=A_{\sigma^{(n)}}^{(n)}$. We will first study asymptotics of $A_{k}^{(n)}$. For this, we use a two-step approximation method close to Section 4 of [15]. Define first

$$
\tilde{A}_{k}^{(n)}=\sum_{i=1}^{k \wedge \tau^{(n)}} \frac{1}{g_{Y_{i-1}^{(n)}}},
$$

obtained replacing the $e_{i}$ 's by their mean,and

$$
\hat{A}_{k}^{(n)}=\frac{1}{C_{0} \Gamma(2-\alpha)} \sum_{i=1}^{k \wedge \tau^{(n)}}\left(Y_{i-1}^{(n)}\right)^{-\alpha},
$$

obtained replacing $g_{b}$ by its equivalent in (6).

Proposition 4.1. We assume that $\rho(t)=C_{0} t^{-\alpha}+O\left(t^{-\alpha+\zeta}\right)$ for some $C_{0}>0$ and $\zeta>1-1 / \alpha$. Then, for any $t<\alpha-1$, we have

$$
n^{\alpha-1} A_{\lfloor n t\rfloor}^{(n)} \stackrel{\mathbb{P}}{\rightarrow} \frac{1}{C_{0} \Gamma(2-\alpha)}\left(\left(1-\frac{t}{\alpha-1}\right)^{1-\alpha}-1\right)
$$

when $n \rightarrow \infty$.

The proof is a straight consequence of Lemma 3.3 with $\eta=\alpha$ and the following Lemmas 4.2 and 4.3 . 
Lemma 4.2. Under the assumptions of Proposition 4.1, we have

$$
n^{\alpha-1}\left(\tilde{A}_{\lfloor n t\rfloor}^{(n)}-\hat{A}_{\lfloor n t\rfloor}^{(n)}\right) \stackrel{\mathbb{P}}{\rightarrow} 0,
$$

when $n \rightarrow \infty$.

Proof. Use (6) to get

$$
\tilde{A}_{\lfloor n t\rfloor}^{(n)}-\hat{A}_{\lfloor n t\rfloor}^{(n)}=\sum_{i=1}^{\lfloor n t\rfloor \wedge \tau^{(n)}}\left(Y_{i-1}^{(n)}\right)^{-\alpha} O\left(\left(Y_{i-1}^{(n)}\right)^{-\min (\zeta, 1)}\right) .
$$

The result then follows from Lemma 3.3 with $\eta=\alpha+\min (\zeta, 1)$.

Lemma 4.3. Under the assumptions of Proposition 4.1, we have

$$
n^{\alpha-1}\left(A_{\lfloor n t\rfloor}^{(n)}-\tilde{A}_{\lfloor n t\rfloor)}^{(n)}\right) \stackrel{\mathbb{P}}{\rightarrow} 0,
$$

when $n \rightarrow \infty$.

Proof. Recall that $\mathcal{Y}=\left(\mathcal{Y}_{k}, k \geq 0\right)$ denotes the filtration generated by $Y$. Conditionally on $\mathcal{Y}$, the random variables $\frac{e_{i}-1}{g_{Y_{i-1}^{(n)}}}$ are independent with zero mean. We deduce that

$$
\begin{aligned}
\mathbb{E}\left[\sup _{t \geq 0}\left(n^{\alpha-1}\left(A_{\lfloor n t\rfloor}^{(n)}-\tilde{A}_{\lfloor n t\rfloor}^{(n)}\right)\right)^{2} \mid \mathcal{Y}\right] & =n^{2 \alpha-2} \mathbb{E}\left[\sup _{t \geq 0}\left(\sum_{i=1}^{\lfloor n t\rfloor \wedge \tau^{(n)}} \frac{e_{i}-1}{g_{Y_{i-1}^{(n)}}}\right)^{2} \mid \mathcal{Y}\right] \\
& \leq 4 n^{2 \alpha-2} \sum_{i=1}^{\lfloor n t\rfloor \wedge \tau^{(n)}}\left(\frac{1}{g_{Y_{i-1}^{(n)}}}\right)^{2},
\end{aligned}
$$

where we used Doob's inequality for the inequality. Thanks to (6) and Lemma 3.3 with $\eta=2 \alpha$, we get the $4 n^{2 \alpha-2} \sum_{i=1}^{\left\lfloor n t \backslash \wedge \tau^{(n)}\right.}\left(\frac{1}{g_{Y_{i-1}^{(n)}}}\right)^{2}$ converges to 0 in probability.

Heuristically, combining Theorem 3.1 and Proposition 4.1, we should get that $n^{\alpha-1} T^{(n)}=$ $n^{\alpha-1} A_{\sigma^{(n)}}^{(n)}$ converges in law to $\frac{1}{C_{0} \Gamma(2-\alpha)}\left((1-\sigma)^{1-\alpha}-1\right)$. This line of proof will be followed in the last section. However, in the next section we will first present another way to prove this result with a method based on the consistency property of exchangeable coalescents. As a first step to this approach, we end this session with a result about small-time behavior of the block-counting process.

Let $R_{t}^{(n)}$ denote the number of blocks of the $n$-coalescent $\Pi^{(n)}$ at time $t$. The initial value $R_{0}^{(n)}$ is $n$. We show that the limit law of the process $R^{(n)}$ is deterministic under a certain time rescaling

Theorem 4.4. We assume that $\rho(t)=C_{0} t^{-\alpha}+O\left(t^{-\alpha+\zeta}\right)$ for some $C_{0}>0$ and $\zeta>1-1 / \alpha$. For any $t_{0}>0, \varepsilon>0$, we have

$$
\mathbb{P}\left(\sup _{0 \leq t \leq t_{0}}\left|n^{-1} R_{t n^{1-\alpha}}^{(n)}-\left(1+C_{0} \Gamma(2-\alpha) t\right)^{-1 /(\alpha-1)}\right|>\varepsilon\right) \rightarrow 0,
$$

when $n \rightarrow \infty$. 
Proof. Let $0<r<\alpha-1$, we have the following relation :

$$
R_{A_{\lfloor n r\rfloor}^{(n)}}^{(n)}=Y_{\lfloor n r\rfloor}^{(n)}=n-\sum_{j=1}^{\lfloor n r\rfloor \wedge \tau^{(n)}} X_{j}^{(n)}
$$

Let $t \in\left[0, t_{0}\right]$, and define

$$
r(t)=(\alpha-1)\left(1-\left(1+C_{0} \Gamma(2-\alpha) t\right)^{-1 /(\alpha-1)}\right),
$$

on $\left[0, t_{0}\right]$. Notice that

$$
\frac{1}{C_{0} \Gamma(2-\alpha)}\left(\left(1-\frac{r(t)}{\alpha-1}\right)^{1-\alpha}-1\right)=t .
$$

Then thanks to Proposition 4.1, $n^{\alpha-1} A_{\lfloor n r(t)\rfloor}^{(n)}$ converges in probability to $t$.

Using the remark at the beginning of the proof in Lemma 3.5, we get the convergence

$$
n^{-1} R_{A_{\lfloor n r(t)\rfloor}^{(n)}}^{(n)}=\frac{Y_{\lfloor n r(t)\rfloor}^{(n)}}{n} \stackrel{\mathbb{P}}{\rightarrow}\left(1-\frac{r(t)}{\alpha-1}\right)=\left(1+C_{0} \Gamma(2-\alpha) t\right)^{-1 /(\alpha-1)},
$$

when $n \rightarrow \infty$. Moreover, since $R_{t}^{(n)}$ is decreasing, then for any $0<\delta<1$,

$$
\lim _{n \rightarrow \infty} \mathbb{P}\left(R_{A_{\lfloor n r(t-\delta t)\rfloor}^{(n)}}^{(n)} \leq R_{t n^{1-\alpha+1}}^{(n)} \leq R_{A_{\lfloor n r(t+\delta t)\rfloor}^{(n)}}^{(n)}\right)=1 .
$$

The constant $\delta$ being arbitrary, we thus obtain the convergence in probability of $n^{-1} R_{t n^{1-\alpha}}^{(n)}$ to $\left(1+C_{0} \Gamma(2-\alpha) t\right)^{-1 /(\alpha-1)}$.

We obtain (24) using again the fact that $R^{(n)}$ is a decreasing process.

In fact, the asymptotic result concerning block counting process of Kingman's coalescent is also valid. The method is almost identical to that employed in the above Theorem. In the context of Kingman's coalescent, we use the same notations $\Pi^{(n)}, A_{i}^{(n)}, R^{(n)}$.

Theorem 4.5. In the setting of the Kingman's coalescent, for any $t_{0}>0, \varepsilon>0$, we have

$$
\mathbb{P}\left(\sup _{0 \leq t \leq t_{0}}\left|n^{-1} R_{t n^{-1}}^{(n)}-(1+t / 2)^{-1}\right|>\varepsilon\right) \rightarrow 0
$$

when $n \rightarrow \infty$.

Remark that this Theorem shows a nice continuity from Beta-coalescent(the process that we consider is more general but contains Beta-coalescent) to Kingman's coalescent., setting $\alpha=2$ in Theorem 4.4 .

Proof. Recall that $A_{i}^{(n)}$ is the time when $i$ th jump is achieved. When $\Pi^{(n)}$ has $b$ individuals at some time $t$, then the process encounters the following coalescence at rate $\left(\begin{array}{l}b \\ 2\end{array}\right)$ where two randomly chosen individuals will be coalesced. $\Pi^{(n)}$ remains 1 when all individuals are coalesced.

For $0<t<1$, we have

$$
A_{\lfloor n t\rfloor}^{(n)}=\sum_{k=n-\lfloor n t\rfloor+1}^{n} \frac{e_{k}}{\left(\begin{array}{l}
k \\
2
\end{array}\right)}
$$


where $e_{i}$ s are i.i.d unit exponential variables. Notice that

$$
\mathbb{E}\left[n A_{\lfloor n t\rfloor}^{(n)}\right]=\sum_{k=n-\lfloor n t\rfloor+1}^{n} \frac{n}{\left(\begin{array}{c}
k \\
2
\end{array}\right)}=2\left(\frac{1}{n-\lfloor n t\rfloor}-\frac{1}{n}\right) n \rightarrow 2\left(\frac{1}{1-t}-1\right),
$$

as $n$ tends to $\infty$. There exist a constant $K>0$, such that,

$$
\operatorname{Var}\left(n A_{\lfloor n t\rfloor}^{(n)}\right)=\sum_{k=n-\lfloor n t\rfloor+1}^{n} n^{2}\left(\frac{1}{\left(\begin{array}{c}
k \\
2
\end{array}\right)}\right)^{2} \leq \frac{K}{n} .
$$

So we deduce that

$$
n A_{\lfloor n t\rfloor}^{(n)} \stackrel{L^{2}}{\rightarrow} 2\left(\frac{1}{1-t}-1\right)=\frac{2 t}{1-t}:=f(t)
$$

as $n$ converges to $\infty$.

We denote by $f^{-1}(t):=t /(t+2)$ the inverse function of $f(t)$.

Similarly, $R^{(n)}$ is decreasing, so

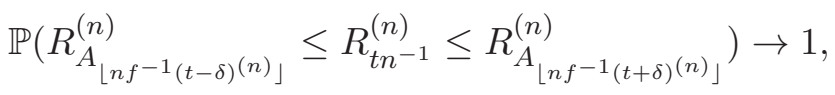

as $n$ tends to $\infty$ for any $0<\delta<t$.

So $\frac{R_{t n-1}^{(n)}}{n}-\frac{R_{\lfloor n f}^{(n)}}{n} \stackrel{d}{\rightarrow} 0$.

Furthermore,

$$
\frac{R_{A_{n f-1}^{(n)}(t)}^{(n)}}{n}=\frac{Y_{\left\lfloor n f^{-1}(t)\right\rfloor}^{(n)}}{n}=\frac{n-\left\lfloor n f^{-1}(t)\right\rfloor}{n} \rightarrow 1-f^{-1}(t)=\frac{1}{1+t / 2},
$$

as $n$ tends to $\infty$. So $\frac{R_{t n-1}^{(n)}}{n} \stackrel{d}{\rightarrow} \frac{1}{1+t / 2}$.

Using again the decreasing property of $R_{t}^{(n)}$, we finish the proof.

\section{The LENGTH OF AN EXTERnAL BRANCH PICKED AT RANDOM}

Dynamics of any exchangeable coalescent with multiple mergers are characterized by rates $\lambda_{b, k}$ which suit a consistent relationship (this is Pitman's structure theorem, see [30], Lemma 18):

$$
\lambda_{b, k}=\lambda_{b+1, k+1}+\lambda_{b+1, k} .
$$

This relationship comes from the fact that $k$ given merging blocks among $b$ can coalesce in two ways while revealing an extra block : either the coalescence event implies the extra block (and then $k+1$ blocks will merge) or not. Thus we get a recursive construction of the $n$-coalescent process $\Pi^{(n)}$.

Let us define $\Pi^{(n, 2)}$ as the coalescent process of individuals labelled from 2 to $n$. Now we consider the individual labelled by 1 . The lineage of this individual can be 'connected' to $\Pi^{(n, 2)}$

- either at any of its jump times, in which case block $\{1\}$ participates to a multiple merger implying at least 3 blocks, and we call this collision "Type 1" (see Figure 1),

- or at any other time to one of the present blocks and then participates to a binary collision, and we call it "Type 2" (see Figure 2). 


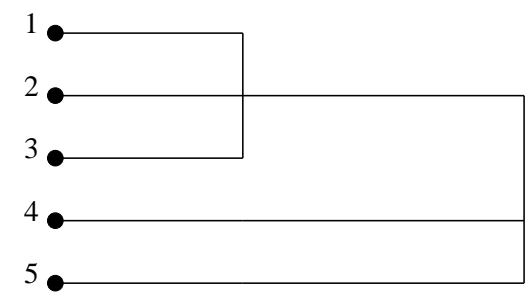

FiguRE 1. $n=5$. Individual 1 is chosen. Type 1: individual 1 encounters a multiple collision.

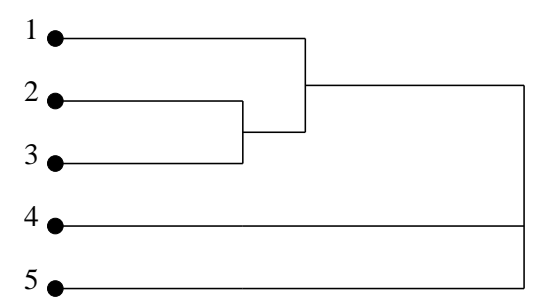

FiguRE 2. $n=5$. Individual 1 is chosen. Type 2: individual 1 encounters a binary collision

From now on, our analysis is conditional on $\Pi^{(n, 2)}$. Between two jump times of $\Pi^{(n, 2)}$, assuming that there are $b$ blocks in $\Pi^{(n, 2)}$, the extra block coalesces at rate $b \lambda_{b+1,2}$. If the extra block remains unconnected just before a coalescence event implying $k$ blocks among $b$, then it will participate to this event with probability

$$
\frac{\int_{0}^{1} x^{k+1}(1-x)^{b-k} \nu(d x) / g_{b}}{\int_{0}^{1} x^{k}(1-x)^{b-k} \nu(d x) / g_{b}}=1-\frac{\lambda_{b+1, k}}{\lambda_{b, k}} .
$$

This equality comes from (27). Let us see how to get the law of $T^{(n)}$, the coalescence time of individual 1 . We define by $R^{(n, 2)}$ the block counting process of $\Pi^{(n, 2)}$. Notice that it has the same law as $R^{(n-1)}$. We introduce

- $T_{c}^{(n)}$ the first jump time of a Poisson process $\eta_{c}^{(n)}$ directed by the measure $\nu_{c}^{(n)}=$ $R_{t}^{(n, 2)} \lambda_{R_{t}^{(n, 2)}+1,2} d t$

- $T_{d}^{(n)}$ the time of the first appearance of 'Head' in the following coin flip, independent of $\eta_{c}^{(n)}$ : at each jump time $t$ of $R_{t}^{(n, 2)}$, we toss a coin, and get 'Head' with probability $1-\frac{\lambda_{R_{t-}^{(n, 2)}+1, R_{t-}^{(n, 2)}-R_{t}^{(n, 2)}+1}}{\lambda_{R_{t-}^{(n, 2)}, R_{t-}^{(n, 2)}-R_{t}^{(n, 2)}+1}}$ and 'Tail' with probability $\frac{\lambda_{R_{t-}^{(n, 2)}+1, R_{t-}^{(n, 2)}-R_{t}^{(n, 2)}+1}}{\lambda_{R_{t-}^{(n, 2)}, R_{t-}^{(n, 2)}-R_{t}^{(n, 2)}+1}}$ (see $\left.(28)\right)$.

Then, conditionally on $\Pi^{(n, 2)}, T^{(n)}$ and $T_{c}^{(n)} \wedge T_{d}^{(n)}$ have the same law.

Remark 5.1. A more formal way to interpret $T^{(n)}$ is as follow. Let $\xi^{(n)}$ be Cox process directed by random measure $\nu_{c}^{(n)}+\nu_{d}^{(n)}$, where $\nu_{d}^{(n)}=\sum_{\{t \text { is a jump time }\}} \frac{\lambda_{R_{t}^{(n, 2)}+1, R_{t-}^{(n, 2)}-R_{t}^{(n, 2)}+1}}{\lambda_{R_{t-}^{(n, 2)}, R_{t-}^{(n, 2)}-R_{t}^{(n, 2)}+1}} \delta_{t}$, and $\delta_{t}$ is the Dirac measure in $t$ (see [25, p.226]). Then $T^{(n)}$ has the same law as the first jump time of $\xi^{(n)}$

Let us now give our main result 
Theorem 5.2. The following convergence holds:

$$
n^{\alpha-1} T^{(n)} \stackrel{d}{\rightarrow} T=\frac{1}{C_{0} \Gamma(2-\alpha)}\left((1-\sigma)^{1-\alpha}-1\right),
$$

for $n \rightarrow \infty$. The density function of $T$ is

$$
f_{T}(t)=\frac{\alpha C_{0} \Gamma(2-\alpha)}{\alpha-1}\left(1+C_{0} \Gamma(2-\alpha) t\right)^{-\frac{\alpha}{\alpha-1}-1}, \quad t \geq 0 .
$$

In particular, in the Beta $(2-\alpha, \alpha)$ case, the density is

$$
f_{T}(t)=\frac{1}{(\alpha-1) \Gamma(\alpha)}\left(1+\frac{t}{\alpha \Gamma(\alpha)}\right)^{-\frac{\alpha}{\alpha-1}-1}, \quad t \geq 0 .
$$

Proof. For the sake of simplicity, we will make the proof only in the $\operatorname{Beta}(2-\alpha, \alpha)$ case. The proof can be extended to the more general case where (5) is satisfied with the details omitted here. In this special case, $C_{0}=(\alpha \Gamma(\alpha) \Gamma(2-\alpha))^{-1}$ and dynamics are given by

$$
\lambda_{b, k}=\frac{B(k-\alpha, b-k+\alpha)}{B(\alpha, 2-\alpha)},
$$

where $B(a, b)$ is a Beta function of parameters $a$ and $b$.

Define $r_{t}^{(n, 2)}$ as the number of jumps of the process $\Pi^{(n, 2)}$ up to time $n^{1-\alpha} t$. It is a straightforward consequence of Proposition 4.1 that

$$
\frac{r_{t}^{(n, 2)}}{n} \stackrel{\mathbb{P}}{\rightarrow} r(t), \quad n \rightarrow \infty
$$

for $t \rightarrow \infty$, where $r(t)$ is defined in (25).

For $i \geq 0$, in the process $\Pi^{(n, 2)}$, we denote by $Y_{i}^{(n, 2)}$ the number of blocks remaining after $i$ jumps which equals 1 from the time all individuals are coalesced to 1 , and $Y_{0}^{(n, 2)}=n-1$. Let $X_{i}^{(n, 2)}=Y_{i-1}^{(n, 2)}-Y_{i}^{(n, 2)}$ be the number of blocks we lose during the $i$ th coalescent event. We write $X_{0}^{(n)}=0$. Notice that $\left(Y^{(n, 2)}, X^{(n, 2)}\right)$ has the same law as $\left(\left(Y^{(n-1)}, X^{(n-1)}\right)\right)$.

Using the description given above, we have

$$
\begin{aligned}
& \mathbb{P}\left(n^{\alpha-1} T^{(n)}>t\right) \\
& =\mathbb{E}\left[\mathbb{P}\left(n^{\alpha-1}\left(T_{c}^{(n)} \wedge T_{d}^{(n)}\right)>t \mid \Pi^{(n, 2)}\right)\right] \\
& =\mathbb{E}\left[\mathbb{P}\left(n^{\alpha-1} T_{c}^{(n)}>t \mid \Pi^{(n, 2)}\right) \mathbb{P}\left(n^{\alpha-1} T_{d}^{(n)}>t \mid \Pi^{(n, 2)}\right)\right] \\
& =\mathbb{E}\left[\exp \left(-\int_{0}^{t} \int_{0}^{1} n^{1-\alpha} R_{s n^{1-\alpha}}^{(n, 2)} x^{2}(1-x)^{R_{s n^{1-\alpha}}^{(n, 2)}-1} \nu(d x) d s\right) \prod_{i=1}^{r_{t}^{(n)}} \frac{\lambda_{1+Y_{i-1}^{(n, 2)}, 1+X_{i}^{(n, 2)}}}{\lambda_{Y_{i-1}^{(n, 2)}, 1+X_{i}^{(n, 2)}}}\right] \\
& =\mathbb{E}\left[\exp \left(-\int_{0}^{t} n^{1-\alpha} R_{s n^{1-\alpha}}^{(n, 2)} \frac{B\left(2-\alpha, R_{s n^{1-\alpha}}^{(n, 2)}+\alpha-1\right)}{B(2-\alpha, \alpha)} d s\right) \prod_{i=1}^{r_{t}^{(n)}} \frac{Y_{i-1}^{(n, 2)}-X_{i}^{(n, 2)}+\alpha-1}{Y_{i-1}^{(n, 2)}}\right] .
\end{aligned}
$$

We decompose the term in the expectation into two parts: the exponential on one side and the product on the other.

Let us first look at the exponential term. Using Stirling's formula we get that, for $0 \leq s \leq t$,

$$
n^{1-\alpha} R_{s n^{1-\alpha}}^{(n, 2)} \frac{B\left(2-\alpha, R_{s n^{1-\alpha}}^{(n, 2)}+\alpha-1\right)}{B(2-\alpha, \alpha)}=n^{1-\alpha} \frac{\left(R_{s n^{1-\alpha}}^{(n, 2)}\right)^{\alpha-1}}{\Gamma(\alpha)}+\left(\frac{R_{s n^{1-\alpha}}^{(n, 2)}}{n}\right)^{\alpha-1} f\left(R_{s n^{1-\alpha}}^{(n, 2)}\right),
$$


where $f=f(t)_{\{t \geq 0\}}$ is a deterministic function which converges to 0 as $t$ converges to $\infty$. The sequence $\left(R_{s n^{1-\alpha}}^{(n, 2)}, n \geq 2\right)$ is decreasing so, thanks to Theorem 4.4, we deduce that $\sup _{0 \leq s \leq t}\left(\frac{R_{s n^{1-\alpha}}^{(n, 2)}}{n}\right)^{\alpha-1} f\left(R_{s n^{1-\alpha}}^{(n, 2)}\right)$ converges in probability to 0 as $n$ tends to $\infty$. Consequently, using again Theorem 4.4, we get that

$$
\exp \left(-\int_{0}^{t} n^{1-\alpha} R_{s n^{1-\alpha}}^{(n, 2)} \frac{B\left(2-\alpha, R_{s n^{1-\alpha}}^{(n, 2)}+\alpha-1\right)}{B(2-\alpha, \alpha)} d s\right) \stackrel{\mathbb{P}}{\rightarrow}\left(1+\frac{t}{\alpha \Gamma(\alpha)}\right)^{-\alpha}, \quad n \rightarrow \infty .
$$

Convergence of the product term is obtained by the same method as in proof of Theorem 3.1 , combined with the convergence in (29). To avoid showing almost the same reasoning, we leave the details to readers. This way, we have

$$
\prod_{i=1}^{r_{t}^{(n, 2)}} \frac{Y_{i-1}^{(n, 2)}-X_{i}^{(n, 2)}+\alpha-1}{Y_{i-1}^{(n, 2)}} \stackrel{\mathbb{P}}{\rightarrow}\left(1+\frac{t}{\alpha \Gamma(\alpha)}\right)^{-\frac{\alpha(2-\alpha)}{\alpha-1}}, \quad n \rightarrow \infty .
$$

The product of (30) and (31) then converges in probability to $\left(1+\frac{t}{\alpha \Gamma(\alpha)}\right)^{-\alpha /(\alpha-1)}$. Since this product is bounded, we get that

$$
\mathbb{P}\left(n^{\alpha-1} T^{(n)}>t\right) \rightarrow\left(1+\frac{t}{\alpha \Gamma(\alpha)}\right)^{-\frac{\alpha}{\alpha-1}}, \quad n \rightarrow \infty .
$$

We achieve the proof.

As a consequence of Theorem 5.2, we can get an asymptotic result on the size of the population at the moment of collision of individual 1.

Corollary 5.3. The following convergence holds :

$$
n^{-1} Y_{\sigma^{(n)}}^{(n)} \stackrel{d}{\rightarrow}\left(1+C_{0} \Gamma(2-\alpha) T\right)^{-1 /(\alpha-1)}=1-\sigma
$$

for $n \rightarrow \infty$. Moreover, the density function of this limit is $\alpha x^{\alpha-1} \mathbf{1}_{\{0 \leq x \leq 1\}}$.

Proof. In terms of block counting process, we have $Y_{\sigma^{(n)}}^{(n)}=R_{T^{(n)}}^{(n)}$. Notice that $R_{T^{(n)}}^{(n)}=$ $R_{n^{1-\alpha}\left(n^{\alpha-1} T^{(n)}\right)}^{(n)}$. Using Theorem 5.2, we known that $n^{\alpha-1} T^{(n)}$ converges in distribution to $T$. Hence, if $t_{0}>0$, we deduce from Theorem 4.4 that

$$
\mathbf{1}_{\left\{n^{\alpha-1} T^{(n)}<t_{0}\right\}} \frac{R_{n^{1-\alpha}\left(n^{\alpha-1} T^{(n)}\right)}^{(n)}}{n} \stackrel{d}{\rightarrow} \mathbf{1}_{\left\{T<t_{0}\right\}}\left(1+C_{0} \Gamma(2-\alpha) T\right)^{-1 /(\alpha-1)} .
$$

This achieves the proof.

\section{An alternative Proof for Theorem 5.2}

In this section, we present an alternative proof for Theorem 5.2 using the convergence results for $\sigma^{(n)}$ from Theorem 3.1. First, we need a stronger version of Proposition 4.1 which gives weak convergence in the path space. Recall that

$$
A_{k}^{(n)}=\sum_{i=1}^{k \wedge \tau^{(n)}} \frac{e_{i}}{g_{Y_{i-1}^{(n)}}}
$$

where the $e_{i}$ 's are independent standard exponential variables. 
Proposition 6.1. We assume that $\rho(t)=C_{0} t^{-\alpha}+O\left(t^{-\alpha+\zeta}\right)$ for some $C_{0}>0$ and $\zeta>1-1 / \alpha$. Then, for any $t<\alpha-1$, we have

$$
\left(n^{\alpha-1} A_{\lfloor n s\rfloor}^{(n)}\right)_{s \leq t} \stackrel{d}{\rightarrow}\left(\frac{1}{C_{0} \Gamma(2-\alpha)}\left(\left(1-\frac{s}{\alpha-1}\right)^{1-\alpha}-1\right)\right)_{s \leq t},
$$

in the sense of convergence in the path space $D[0, t]$ for $n \rightarrow \infty$.

Proof. Note that Theorem 4.1 states

$$
n^{\alpha-1} A_{\lfloor n s\rfloor}^{(n)} \stackrel{\mathbb{P}}{\rightarrow}\left(\frac{1}{C_{0} \Gamma(2-\alpha)}\left(\left(1-\frac{s}{\alpha-1}\right)^{1-\alpha}-1\right)\right),
$$

for $0<s<\alpha-1$ and $n \rightarrow \infty$. So for every fixed $s \in[0, t]$, we have pointwise convergence in probability in (32). This implies weak convergence of all finite dimensional distributions due to the subsequence criterion for weak convergence. In order to show weak convergence in the path space, we will show tightness for the distributions from (32). Since the limit process is continuous, it suffices to show that the condition (i) of [7, Theorem 7.3] and condition (7.12) from [7, Corollary 7.4] are fulfilled (see [7, Corollary 13.4]). For the present processes, these conditions translate to showing that for every $\epsilon>0$ and $\eta>0$,

(i) there exists $a>0$ s.t. $P\left(n^{\alpha-1} A_{\lfloor 0\rfloor}^{(n)} \geq a\right) \leq \eta$ for $n$ big enough and

(ii) there exists a $0<\delta<1$ so that

$$
\delta^{-1} P\left(n^{\alpha-1}\left(A_{\left\lfloor n \cdot \min \left(t_{1}+\delta, t\right)\right\rfloor}^{(n)}-A_{\left\lfloor n \cdot t_{1}\right\rfloor}^{(n)}\right) \geq \epsilon\right) \leq \eta,
$$

for $n$ big enough and any $t_{1} \in[0, t]$.

Condition $(i)$ is trivially fulfilled, for condition (ii) we can use Theorem 4.1 to show that for $n \rightarrow \infty$,

$$
P\left(n^{\alpha-1}\left(A_{\left\lfloor n \cdot \min \left(t_{1}+\delta, t\right)\right\rfloor}^{(n)}-A_{\left\lfloor n \cdot t_{1}\right\rfloor}^{(n)}\right) \geq \epsilon\right) \rightarrow P\left(f\left(\min \left(t_{1}+\delta, t\right)\right)-f\left(t_{1}\right) \geq \epsilon\right),
$$

where $f(s):=\frac{1}{C_{0} \Gamma(2-\alpha)}\left(\left(1-\frac{s}{\alpha-1}\right)^{1-\alpha}-1\right)$. Note that $P\left(f\left(\min \left(t_{1}+\delta, t\right)\right)-f\left(t_{1}\right) \geq \epsilon\right) \leq$ $P(f(t)-f(t-\delta) \geq \epsilon) \in\{0,1\}$. Since $f$ is continuous, you can now choose $\delta$ small enough that $f(t)-f(t-\delta)<\epsilon$ and then $n$ big enough to fulfill (ii). Thus, we have shown tightness of the distributions in (32) which establishes the desired weak convergence

Now we come to the alternative proof of Theorem 5.2.

Alternative proof of Theorem 5.2. Fix $t \in[0, \alpha-1)$. We have

$$
\left(\sigma^{(n)} /(n(\alpha-1)),\left(n^{\alpha-1} A_{\lfloor n s\rfloor}^{(n)}\right)_{s \leq t}\right) \stackrel{d}{\rightarrow}\left(\sigma,\left(\frac{1}{C_{0} \Gamma(2-\alpha)}\left(\left(1-\frac{s}{\alpha-1}\right)^{1-\alpha}-1\right)\right)_{s \leq t}\right),
$$

for $n \rightarrow \infty$. Due to Skorohod-coupling, we can assume that this convergence also holds almost surely. Since $s \mapsto\left(\frac{1}{C_{0} \Gamma(2-\alpha)}\left(\left(1-\frac{s}{\alpha-1}\right)^{1-\alpha}-1\right)\right)$ is continuous on $[0, t]$, the almost sure convergence of $\left(n^{\alpha-1} A_{\lfloor n t\rfloor}^{(n)}\right)_{s \leq t}$ in $D[0, t]$ is even almost sure uniform convergence on $[0, t]$ (see $\left[7\right.$, p. 124]). For any series $\left(x_{n}\right)_{n \in \mathbb{N}}$ on $[0, \mathrm{t}]$ with $x_{n} \rightarrow x$, we thus have

$$
n^{\alpha-1} A_{\left\lfloor n x_{n}\right\rfloor}^{(n)} \rightarrow \frac{1}{C_{0} \Gamma(2-\alpha)}\left(\left(1-\frac{x}{\alpha-1}\right)^{1-\alpha}-1\right)
$$

almost surely for $n \rightarrow \infty$. The only problem left is that $\sigma^{(n)}, \sigma$ may take values in $[0, \alpha-1)$ and not only in some subset $[0, t]$. To remedy this, note that if we restrict all random variables on $\left\{\sigma \leq \alpha-1-\frac{2}{k}\right\}$ for $k \in \mathbb{N}$, we have $\sigma^{(n)}(\omega) / n \leq \alpha-1-\frac{1}{k}$ for $n=n(\omega)$ big enough 
for almost all $\omega \in\left\{\sigma \leq \alpha-1-\frac{1}{k}\right\}$. Thus, by using the Skorohod-coupling for the series $\left(\sigma^{(n)} /(n(\alpha-1)),\left(n^{\alpha-1} A_{\lfloor n s\rfloor}^{(n)}\right)_{s \leq \alpha-1-\frac{1}{k}}\right)$, we have

$$
n^{\alpha-1} A_{\left\lfloor n \cdot \sigma^{(n)} / n\right\rfloor}^{(n)} \stackrel{d}{\rightarrow} \frac{1}{C_{0} \Gamma(2-\alpha)}\left(\left(1-\frac{\sigma}{\alpha-1}\right)^{1-\alpha}-1\right),
$$

almost surely on $\left\{\sigma \leq \alpha-1-\frac{2}{k}\right\}$ for the coupled versions of these random variables (note that $\left.\sigma^{(n)} \leq \tau^{(n)}\right)$. Since $\sigma$ is Beta-distributed, we have, for $k \rightarrow \infty$,

$$
P\left(\left\{n^{\alpha-1} A_{\left\lfloor n \cdot \sigma^{(n)} / n\right\rfloor}^{(n)} \in \cdot\right\} \cap\left\{\sigma \leq \alpha-1-\frac{2}{k}\right\}\right) \sim P\left(n^{\alpha-1} A_{\left\lfloor n \cdot \sigma^{(n)} / n\right\rfloor}^{(n)} \in \cdot\right) .
$$

This shows

$$
n^{\alpha-1} T^{(n)}=n^{\alpha-1} A_{\sigma^{(n)}}^{(n)} \stackrel{d}{\rightarrow} \frac{1}{C_{0} \Gamma(2-\alpha)}\left(\left(1-\frac{\sigma}{\alpha-1}\right)^{1-\alpha}-1\right)
$$

The only thing left to prove is that $T$ has density $f_{T}$. This is done by computing the distribution function

$$
\begin{aligned}
& \mathbb{P}\left(\frac{1}{C_{0} \Gamma(2-\alpha)}\left(\left(1-\frac{\sigma}{\alpha-1}\right)^{1-\alpha}-1\right) \leq t\right) \\
= & \mathbb{P}\left(\sigma \leq 1-\left(1+C_{0} \Gamma(2-\alpha) t\right)^{\frac{1}{\alpha-1}}\right) \\
= & \int_{0}^{1-\left(1+C_{0} \Gamma(2-\alpha) t\right)^{\frac{1}{\alpha-1}}} \alpha(1-x)^{\alpha} d x \\
= & 1-\left(1+C_{0} \Gamma(2-\alpha) t\right)^{-\frac{\alpha}{\alpha-1}} .
\end{aligned}
$$

and finally by differentiating.

Acknowledgments. Jean-Stéphane Dhersin and Linglong Yuan benefited from the support of the "Agence Nationale de la Recherche": ANR MANEGE (ANR-09-BLAN-0215). Arno Siri-Jégousse and Fabian Freund would like to thank the Haussdorf Research Institute for Mathematics for its support.

Fabian Freund would like to thank Luca Ferretti for a discussion concerning the interpretation of $\sigma^{(n)}$.

\section{REFERENCES}

[1] E. Árnason. Mitochondrial cytochrome b variation in the high-fecundity atlantic cod: trans-atlantic clines and shallow gene genealogy. Genetics., 15:166: 1871-1885, 2004.

[2] J. Berestycki, N. Berestycki, and V. Limic. The lambda-coalescent speed of coming down from infinity, 2008.

[3] J. Berestycki, N. Berestycki, and J. Schweinsberg. Beta-coalescents and continuous stable random trees. Ann. Probab., 35(5):1835-1887, 2007.

[4] J. Berestycki, N. Berestycki, and J. Schweinsberg. Small-time behavior of beta coalescents. Ann. Inst. H. Poincaré Probab. Statist., 44:214, 2008.

[5] J. Bertoin. Lévy processes, volume 121 of Cambridge Tracts in Mathematics. Cambridge University Press, Cambridge, 1996.

[6] J. Bertoin and J.-F. Le Gall. Stochastic flows associated to coalescent processes. III. Limit theorems. Illinois J. Math., 50(1-4):147-181 (electronic), 2006.

[7] P. Billingsley. Convergence of probability measures. Wiley Series in Probability and Statistics: Probability and Statistics. John Wiley \&amp; Sons Inc., New York, second edition, 1999. A Wiley-Interscience Publication.

[8] M. Birkner and J. Blath. Computing likelihoods for coalescents with multiple collisions in the infinitelymany-sites model. Journal of Mathematical Biology, 2008. 
[9] M. Birkner, J. Blath, M. Capaldo, A. Etheridge, M. Möhle, J. Schweinsberg, and A. Wakolbinger. Alphastable branching and beta-coalescents. Electron. J. Probab., 10:no. 9, 303-325 (electronic), 2005.

[10] M. G. B. Blum and O. François. Minimal clade size and external branch length under the neutral coalescent. Adv. in Appl. Probab., 37(3):647-662, 2005.

[11] E. Bolthausen and A. Sznitman. On ruelle's probability cascades and an abstract cavity method. Communications in mathematical physics, 197(2):247-276, 1998.

[12] J. Boom, E. Boulding, and A. Beckenbach. Mitochondrial DNA variation in introduced populations of Pacific oyster, Crassostrea gigas, in British Columbia. Canadian journal of fisheries and aquatic sciences(Print), 51(7):1608-1614, 1994.

[13] A. Bovier and I. Kurkova. Much ado about derrida's grem. Spin glasses, pages 81-115, 2007.

[14] A. Caliebe, R. Neininger, M. Krawczak, and U. Roesler. On the length distribution of external branches in coalescence trees: genetic diversity within species. Theoretical Population Biology, 72(2):245-252, 2007.

[15] J.-F. Delmas, J.-S. Dhersin, and A. Siri-Jégousse. Asymptotic results on the length of coalescent trees. Ann. Appl. Probab., 18(2):997-1025, 2008.

[16] B. Eldon and J. Wakeley. Coalescent processes when the distribution of offspring number among individuals is highly skewed. Genetics, 172:2621-2633, 2006.

[17] F. Freund and M. Möhle. On the time back to the most recent common ancestor and the external branch length of the Bolthausen-Sznitman coalescent. Markov Process. Related Fields, 15(3):387-416, 2009.

[18] Y. Fu and W. Li. Statistical tests of neutrality of mutations. Genetics, 133:693-709, 1993.

[19] A. Gnedin, A. Iksanov, and M. Möhle. On asymptotics of exchangeable coalescents with multiple collisions. J. Appl. Probab., 45(4):1186-1195, 2008.

[20] A. Gnedin and Y. Yakubovich. On the number of collisions in $\Lambda$-coalescents. Electron. J. Probab., 12:no. 56, 1547-1567 (electronic), 2007.

[21] C. Goldschmidt and J. B. Martin. Random recursive trees and the Bolthausen-Sznitman coalescent. Electron. J. Probab., 10:no. 21, 718-745 (electronic), 2005.

[22] D. Hedgecock. Does variance in reproductive success limit effective population sizes of marine organisms? In Genetics and Evolution of Aquatic Organisms, pages 1222-1344. Chapman and Hall, London, 1994.

[23] A. Iksanov and M. Möhle. On the number of jumps of random walks with a barrier. Advances in Applied Probability, 40(1):206-228, 2008.

[24] S. Janson and G. Kersting. The external lengths in kingman's coalescent, 2011.

[25] O. Kallenberg. Foundations of modern probability. Probability and its Applications (New York). SpringerVerlag, New York, second edition, 2002.

[26] J. F. C. Kingman. The coalescent. Stochastic Process. Appl., 13(3):235-248, 1982.

[27] J. F. C. Kingman. On the genealogy of large populations. J. Appl. Probab., (Special Vol. 19A):27-43, 1982. Essays in statistical science.

[28] M. Möhle. Total variation distances and rates of convergence for ancestral coalescent processes in exchangeable population models. Adv. in Appl. Probab., 32(4):983-993, 2000.

[29] M. Möhle. Asymptotic results for coalescent processes without proper frequencies and applications to the two-parameter Poisson-Dirichlet coalescent. Stochastic Process. Appl., 120(11):2159-2173, 2010.

[30] J. Pitman. Coalescents with multiple collisions. Ann. Probab., 27(4):1870-1902, 1999.

[31] S. Sagitov. The general coalescent with asynchronous mergers of ancestral lines. J. Appl. Probab., 36(4):1116-1125, 1999.

[32] J. Schweinsberg. Coalescent processes obtained from supercritical Galton-Watson processes. Stochastic Process. Appl., 106(1):107-139, 2003. 
Département de Mathématiques, Université Paris 13, 99 AV. J-B. Clément, F-93430 VilletaNEUSE, FranCE

E-mail address: dhersin@math.univ-paris13.fr

Crop Plant Biodiversity and Breeding Informatics Group (350B), Institute of Plant Breeding, Seed Science and Population Genetics, University of Hohenheim, Fruwirthstrasse 21, 70599 Stuttgart, Germany

E-mail address: ffreund@uni-hohenheim.de

Cimat, A.C., Guanajuato, Mexico

E-mail address: arno@cimat.mx

Département de Mathématiques, Université Paris 13, 99 av. J-B. Clément, F-93430 VilletaNEUSE, France

E-mail address: yuan@math.univ-paris13.fr 